\title{
The importance of the intrauterine environment in shaping the human neonatal epigenome
}

\author{
"...mounting evidence [implicates] the time in utero in the fetal 'programming' of \\ human diseases of later life."
}

\section{KEYWORDS: DNA methylation $\approx$ environment $\approx$ epigenetics $\approx$ fetal programming - in utero}

Epigenetic events are an essential mediator of cellular differentiation and, therefore, development in vertebrates. However, the underlying dynamism and responsiveness to subtle subcellular cues that are a hallmark of epigenetic change during development, also renders epigenetic profiles susceptible to external environmental influence, with the potential to 'program' the underlying genome towards an altered phenotype in later life.

\section{The period in utero: a complex \& dependent environment}

For decades it has been postulated that the period in utero may be critical in modifying the risk of adult-onset disease, although only recently has epigenetic change emerged as the likely mediator of such effects. Animal studies have demonstrated conclusively that the environment can shape the developmental phenotype (often stably), particularly during the intrauterine period when it demonstrates the greatest 'plasticity' [1]. The concept of plasticity refers to the potential for multiple phenotypes to arise from a single genotype, potentially (but not always) in response to specific environmental influence. The importance of this for human health is well documented, with mounting evidence implicating the time in utero in the 'programming' of human diseases of later life [1]. The capacity for healthy development in the face of plasticity, a process termed canalization by Conrad Waddington, has long been established [2]. However, the role of in utero-acquired epigenetic variation in this process, the relationship between specific environmental exposures and epigenetic variation, the degree to which different tissues show varying sensitivities to environmental influence and the extent to which this interaction is sensitive to underlying genetic, temporal and stochastic influences, remains largely unknown. Similarly, it remains unclear whether there is a constant 'accumulation' of environmentally mediated epigenetic change or whether specific developmental windows are particularly sensitive. It is intuitive to think the very early environment is particularly sensitive. The emerging picture in humans is one of a complex interplay of factors that combine during early human development to shape the newborn epigenome and potentially influence later health.

\section{Maternal effects}

Animal studies have provided clear evidence for a role of maternal environmental factors in modulating the epigenetic profile of progeny. This has been extensively reviewed elsewhere [3], although the interpretation and significance of the majority of findings is difficult owing to a general lack of replication, small sample size, small effect size or lack of any functional interrogation of the effects of observable epigenetic change between exposure and control groups.

The situation in humans is even less clear given the inherent problems associated with unraveling contributions of genetic and environmental origin to epigenetic change. This is further complicated by the fact that many maternal-associated factors, such as diet, stress, alcohol intake and exposure to endocrine disruptors or pesticides, all implicated in changing the developing epigenetic profile (reviewed in [4]), are extremely difficult to measure accurately in a human setting. Even studies investigating the influence of more readily measurable factors, such as maternal BMI and the use of assisted reproductive technologies, have suffered from a lack of standardized measures, conflicting findings and/or lack of replication.

The most robust findings to date have linked maternal smoking to specific changes in DNA

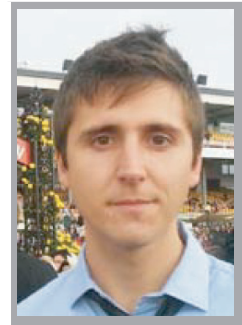

Boris Novakovic Cancer, Disease \& Developmental Epigenetics, Murdoch Childrens Research Institute, Royal Children's Hospital \& Department of Paediatrics, University of Melbourne, Melbourne, Victoria 3052, Australia

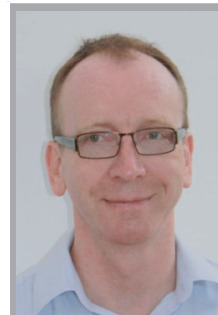

Richard Saffery

Author for correspondence: Cancer, Disease \& Developmental Epigenetics, Murdoch Childrens Research Institute, Royal Children's Hospital \& Department of Paediatrics. University of Melbourne, Melbourne, Victoria 3052, Australia richard.saffery@mcri.edu.au

Future : Medicine part of 
methylation in a large cohort of pregnancies [5]. Consistent changes in genes implicated in detoxification were reported, some of which replicated findings in nonpregnant adult smokers. The postnatal stability of such clearly defined environmentally induced epigenetic change warrants urgent investigation.

\section{Fetal programming, developmental origins of health \& disease \& long-term epigenetic change}

The term 'fetal programming' was first coined in 1979 [6] to describe the long-term effect of the intrauterine environment on offspring. Barker and colleagues further developed this idea in the early 1990s in response to an observed negative association between death from cardiovascular diseases in adults and birth weight in a UK cohort [7-9]. The related developmental origins of the health and disease hypothesis, is supported by a large number of studies in animals and fewer, largely observational studies, in humans $[10,11]$. It is not surprising that epigenetic variation acquired in utero has emerged as a prime candidate for mediating such long-term 'programming' effects.

Epigenetic response to in utero environmental exposure can manifest in several ways. First, as an alteration in developmental processes early in life in the absence of long-lasting epigenetically induced changes (epigenetic 'legacy'). This may or may not be associated with short- or longerterm phenotypic change. Second, as a stable 'response' of the developing pregnancy to a specific environment. This may, or may not, be an adaptation to a 'perceived' postnatal environment related to the in utero exposure. Finally, as a 'neutral' stable epigenetic change at birth with the capacity to modify the later phenotype, independently of the processes (or exposure) that led to the original epigenetic change.

\section{Tissue-specific epigenetic response: 'dosage' effects or tissue-specific adaptation?}

It is becoming increasingly clear that the effects of the in utero environment in shaping the epigenome may be tissue specific. There are several explanations for this observation, including the differential 'dosage' of individual tissues to an environmental exposure associated with fetal blood flow. It is logical to assume that the placenta, as the major interface between maternal and fetal circulations, would receive the maximal 'dose' in such a scenario, although little evidence for an exacerbation of any environmental effect on epigenetic markers in the placenta relative to other tissues has emerged. An alternative explanation involves a tissue-specific 'response' or 'adaptation' to environmental exposures. However, direct evidence to support this alternative viewpoint is also currently lacking. At present, it is unclear how much of the resulting 'adaptation' of the developing pregnancy to a perceived external environment confers a developmental advantage postnatally, and how much is simply a response to changing environmental influence, conferring little advantage or potentially, disadvantage. In either instance, the observed tissue-specific variation in an epigenetic profile at birth in response to in utero environmental exposures has profound implications for monitoring the effects of such exposures using readily obtained tissues collected at birth or early in life.

\section{"It is not surprising that epigenetic variation acquired in utero has emerged as a prime candidate for mediating such long-term 'programming' effects."}

\section{Genetics as a modifier of environmentally induced epigenetic change}

Emerging evidence in twins supports a heritable contribution to overall DNA methylation profiles, albeit low relative to many other phenotypes [12]. Nevertheless, the underlying DNA sequence is clearly important in determining epigenetic status in several loci at birth [13] and as such, may play a role in how the developing pregnancy responds epigenetically to in utero environmental influences. Certain genomic regions/features may be more susceptible to both environmentally and genetically induced epigenetic disruption/variability during early development.

\section{Stochasticity}

There are no examples in nature of completely identical organisms arising from a single genetic blueprint. Whether at the scale of gross phenotypic difference, as commonly seen in human monozygotic twins, or at the molecular level (i.e., the number of ribosomes in a cell), the complexity of even the simplest forms of life ensures that no two organisms are exactly identical. This inherent variation underpins all evolution.

The 'intangible variation' associated with most phenotypes, first described by Gartner [14], can equally be observed in the spectrum of epigenetic variation at many loci in genetically identical 
mammals. Best exemplified by elegant studies at metastable epialleles, such as the agouti-viable yellow $(A[v y])$ mouse model $[15,16]$, stochastic variation is likely to arise as a direct consequence of the generally imperfect mechanisms involved in the establishment and stability of epigenetic marks [17]. Teasing out the relative contributions of environmental and stochastic variation is currently not possible as even genetically identical individuals carried by the same mother experience differing in utero environments associated with variable nutrient 'supply-line' factors (e.g., implantation site, placenta vascularization and cord placement) that are established during early development. Recent data in newborn monozygous twins highlights these effects, with nonshared environmental/stochastic factors clearly contributing to newborn variation in epigenetic profiles $[13,18]$.

More recently, the propensity of the epigenome to show probabilistic (stochastic) variation, in addition to that arising directly in response to environmental exposure, has itself been proposed to be an evolutionary adaption, maximizing the capacity of some, within a population of individuals, to respond to short-term alterations in the environment independently of genetic change. To quote Andy Feinberg, "In mathematical terms, this means that noise is not just a constant term to add to the equation but is itself a function of the developmental landscape"[19].

\section{"Teasing out the relative contributions of environmental and stochastic variation is currently not possible as even genetically identical individuals carried by the same mother experience differing in utero environments..."}

In many ways the proposed hard-wired capacity for epigenetic variability parallels the now established phenomenon of hypervariability in the genetic blueprint through copy number variation. However, whereas the latter likely confers evolutionary advantage to a small number of individuals in an altered environment during evolution, epigenetic variation due to stochasticity is more likely to be normally distributed within a population, thereby conferring a potential advantage to a larger proportion of an exposed population. Given the multitude of developmental levels at which stochastic noise may arise, it is not surprising that the overall effects of this 'third component' to variation might be anticipated to be more evident in complex as opposed to simpler organisms.

\section{Transgenerational modification of environmental responsiveness}

DNA methylation is highly dynamic during early embryogenesis, but contrary to popular belief, is not completely erased during very early development or gametogenesis [20-23]. The mechanisms underlying transgenerational effects are likely to vary according to whether the effect is mediated by gametic transmission or is independent thereof. A recent study examining the DNA methylation remodeling that takes place during gametogenesis in mouse germ cells clearly highlights the fact that epigenetic marks can persist in gametes, particularly in genomic regions that are associated with repetitive elements [24]. Such provocative findings represent a feasible route for the previously described phenomenon of transgenerational epigenetic inheritance [25], although to date, no compelling examples of such inheritance have been documented.

\section{Conclusion}

The field of early developmental epigenetics in humans remains in its infancy and although animal studies continue to pave the way in providing insights as to the dynamic regulation of the developing epigenome in utero, there are many facets of both early development and epigenetic profile that differentiate human pregnancies from those of most animal models. As such, direct human studies are essential. Developing a complete understanding of the interaction between genetic, environmental and stochastic variables in shaping the human epigenome in different tissues prior to birth will be problematic but will be greatly enhanced by the recent advent of large longitudinal birth cohorts with a detailed collection of environmental and other data, multiple biospecimens [26,27] and the evergrowing technical advances in characterization of the epigenetic landscape.

Financial \& competing interests disclosure

$R$ Saffery is supported by a National Health and Medical Research Council (NHMRC) Senior Research Fellowship and B Novakovic is supported by a NHMRC Dora Lush postgraduate scholarship. Both authors are supported by the Victorian Government's Operational Infrastructure Support Program. The authors have no other relevant affiliations or financial involvement with any organization or entity with a financial interest in or financial conflict with the subject matter or materials discussed in the manuscript apart from those disclosed.

No writing assistance was utilized in the production of this manuscript. 


\section{References}

1 Gluckman PD, Hanson MA, Beedle AS. Early life events and their consequences for later disease: a life history and evolutionary perspective. Am. J. Hum. Biol. 19(1), 1-19 (2007)

2 Waddington $\mathrm{CH}$. Canalization of development and genetic assimilation of acquired characters. Nature 183(4676), 1654-1655 (1959).

3 Sebert S, Sharkey D, Budge H, Symonds ME. The early programming of metabolic health: is epigenetic setting the missing link? Am. J. Clin. Nutr. 94(Suppl. 6), S1953-S1958 (2011).

4 Hogg K, Price EM, Hanna CW, Robinson WP. Prenatal and perinatal environmental influences on the human fetal and placental epigenome. Clin. Pharmacol. Ther. 92(6), 716-726 (2012).

5 Joubert BR Håberg SE, Nilsen RM et al. 450K Epigenome-wide scan identifies differential DNA methylation in newborns related to maternal smoking during pregnancy. Environ. Health Perspect. 120(10), 1425-1431 (2012).

6 Koldovsky O. Development of sucrase activity: effect of maternal hormonal status and fetal programming of jejuno-ileal differences. Ciba Found. Symp. (70), 147-168 (1979).

7 Barker DJ, Bull AR, Osmond C, Simmonds SJ. Fetal and placental size and risk of hypertension in adult life. $B M J$ 301(6746), 259-262 (1990).

8 Barker DJ, Osmond C, Simmonds SJ, Wield GA. The relation of small head circumference and thinness at birth to death from cardiovascular disease in adult life. $B M J$ 306(6875), 422-426 (1993).

9 Osmond C, Barker DJ. Ischaemic heart disease in England and Wales around the year
2000. J. Epidemiol. Community Health 45(1), 71-72 (1991).

10 Barker DJ, Winter PD, Osmond C, Margetts B, Simmonds SJ. Weight in infancy and death from ischaemic heart disease. Lancet 2(8663), 577-580 (1989).

11 Roseboom TJ, van der Meulen JH, Ravelli AC, Osmond C, Barker DJ, Bleker OP. Effects of prenatal exposure to the Dutch famine on adult disease in later life: an overview. Mol. Cell. Endocrinol. 185(1-2), 93-98 (2001).

12 Bell JT, Saffery R. The value of twins in epigenetic epidemiology. Int. J. Epidemiol. 41(1), 140-150 (2012).

13 Gordon L, Joo JE, Powell JE et al. Neonatal DNA methylation profile in human twins is specified by a complex interplay between intrauterine environmental and genetic factors, subject to tissue-specific influence. Genome Res. 22(8), 1395-1406 (2012).

14 Gartner K. 2 new aspects in laboratory animal science: "intangible variance" and population biology. Dtsch. Tierarztl. Wochenschr. 89(8), 318-322 (1982).

15 Morgan HD, Sutherland HG, Martin DI, Whitelaw E. Epigenetic inheritance at the agouti locus in the mouse. Nat. Genet. 23(3), 314-318 (1999).

16 Waterland RA, Jirtle RL. Transposable elements: targets for early nutritional effects on epigenetic gene regulation. Mol. Cell. Biol. 23(15), 5293-5300 (2003).

17 Blewitt ME, Chong S, Whitelaw E. How the mouse got its spots. Trends Genet. 20(11), 550-554 (2004).

18 Ollikainen M, Smith KR, Joo EJ et al. DNA methylation analysis of multiple tissues from newborn twins reveals both genetic and intrauterine components to variation in the human neonatal epigenome. Hum. Mol. Genet. 19(21), 4176-4188 (2010).
19 Pujadas E, Feinberg AP. Regulated noise in the epigenetic landscape of development and disease. Cell 148(6), 1123-1131 (2012).

20 Fan S, Zhang X. CpG island methylation pattern in different human tissues and its correlation with gene expression. Biochem. Biophys. Res. Commun. 383(4), 421-425 (2009).

21 Flanagan JM, Popendikyte V, Pozdniakovaite $\mathrm{N}$ et al. Intra- and interindividual epigenetic variation in human germ cells. Am. J. Hum. Genet. 79(1), 67-84 (2006).

22 Rakyan VK, Chong S, Champ ME et al. Transgenerational inheritance of epigenetic states at the murine $\mathrm{Axin}(\mathrm{Fu})$ allele occurs after maternal and paternal transmission. Proc. Natl Acad. Sci. USA 100(5), 2538-2543 (2003).

23 Trasler JM. Epigenetics in spermatogenesis. Mol. Cell. Endocrinol. 306(1-2), 33-36 (2009).

24 Guibert S, Forné T, Weber M. Global profiling of DNA methylation erasure in mouse primordial germ cells. Genome Res. 22(4), 633-641 (2012).

25 Okada Y, Yamagata K, Hong K, Wakayama T, Zhang Y. A role for the elongator complex in zygotic paternal genome demethylation. Nature 463(7280), 554-558 (2010).

26 Tao FB, Hao JH, Huang K et al. Cohort profile: the China-Anhui birth cohort study. Int. J. Epidemiol. doi:10.1093/ije/dys085 (2012) (Epub ahead of print).

27 Jaddoe VW, Mackenbach JP, Moll HA et al. The generation R study: design and cohort profile. Eur. J. Epidemiol. 21(6), 475-484 (2006). 